\title{
Rotationally Ordered Distribution of Ions in a Melt Simulated by Molecular Dynamics
}

\author{
B. Steffen and R. Hosemann \\ Teilinstitut für Strukturforschung am Fritz-Haber-Institut \\ der Max-Planck-Gesellschaft, Berlin-Dahlem \\ K. Heinzinger and L. Schäfer \\ Max-Planck-Institut für Chemie, Mainz
}

(Z. Naturforsch. 32a, 1426-1432 [1977]; received September 3, 1977)

\begin{abstract}
A mean threedimensional density distribution is determined by comparing the surroundings of all atoms of a melt. Contrary to the definition of the pair distribution function, the surroundings of the different atoms are allowed to rotate against each other before being added up.

The rotationally ordered distribution (ROD) function is the sum of the surroundings of all atoms rotated against each other until they give maximum overlap. An investigation of molecular dynamies results for molten $\mathrm{KCl}$ gives 6 nearest neighbourpeaks in a distorted cubic primitive arrangement.

If the $\mathrm{K}^{+}$or $\mathrm{Cl}^{-}$-ions are considered separately, the surrounding gives 12 nearest neighbourpeaks in a thoroughly distorted face centered cubic arrangement. These results confirm the interpretation of the structure of the melt as a paracrystalline distorted lattice and the results of an earlier threedimensional analysis of the structure of molten leaci.
\end{abstract}

\section{Introduction}

The structure factor of liquids, which can be determined from scattering experiments, is always a spherically symmetric function. Its FourierTransform is the pair distribution function $g(r)$

$$
g(r)=\frac{1}{4 \pi r^{2} N \varrho_{0}} \sum_{\mu}^{N} \sum_{\nu \neq \mu}^{N} P\left(r-r_{\mu \nu}\right) .
$$

$r_{\mu \nu}=\left|\boldsymbol{x}_{\mu v}\right|=\left|\boldsymbol{x}_{v}-\boldsymbol{x}_{\mu}\right|$, where $\boldsymbol{x}_{v}$ and $\boldsymbol{x}_{\mu}$ are the positions of the molecules in the liquid at a certain moment. $P(a)$ is a point function ${ }^{1}$ of the argument $a$ with a finite value for $a=0$ and an integral value of one:

$$
\int P(a) \mathrm{d} a=1 .
$$

$\varrho_{0}$ is the mean number density and $N$ a sufficiently large number of molecules.

Generally $g(r)$ is the normalized convolution square of the atomic density distribution

$$
\begin{aligned}
& \varrho(\boldsymbol{x})=\sum_{\nu}^{N} P\left(\boldsymbol{x}-\boldsymbol{x}_{v}\right), \\
& \text { so that } \quad g(\boldsymbol{x})=\frac{1}{N \varrho_{0}} \sum_{\mu}^{N} G_{\mu}(\boldsymbol{x}) \\
& \text { with } \quad G_{\mu}(\boldsymbol{x})=\sum_{\nu \neq \mu}^{N} P\left(\boldsymbol{x}-\boldsymbol{x}_{\mu v}\right) .
\end{aligned}
$$

Reprint requests to Dr. B. Steffen, Fritz-Haber-Institut der Max-Planck-Gesellschaft, Faradayweg 4-6, 1000 Berlin 33/Dahlem.
The functions $G_{\mu}(\boldsymbol{x})$ represent atomic density distributions with the origin of the coordinate system at atom $\mu$.

Apparently each $G_{\mu}$ describes a nonspherical distribution of mass points at any instantaneous time, and one can define an orientation for each $G_{\mu}$ and fix a coordinate system to it. The reason why $g(\boldsymbol{x})$ is spherically symmetric for liquids is the same as for crystalline powders: The position vectors of the atoms relative to atom $\alpha$ have other directions than the position vectors of the atoms relative to atom $\beta$, if the distance between $\alpha$ and $\beta$ is big enough. Therefore the sum (4) will contain position vectors with modulus $r$ in all possible directions with equal probability. Thus

$g(r)=\langle g(\boldsymbol{x})\rangle_{\vartheta . \varphi}:=\frac{1}{4 \pi r^{2}} \int_{0}^{\pi} \int_{0}^{2 \pi} g(\boldsymbol{x}) r^{2} \sin \vartheta \mathrm{d} \vartheta \mathrm{d} \varphi$

and

$$
\langle g(\boldsymbol{x})\rangle_{\vartheta}, \varphi=\frac{1}{N \varrho_{0}} \sum_{\mu}^{N}\left\langle G_{\mu}(\boldsymbol{x})\right\rangle_{\vartheta}, \varphi .
$$

For the following we introduce some conventions in order to simplify the description of what we are doing.

1. "Orientation of $G_{\mu}(\boldsymbol{x})$ " means: An arbitrary choice of a coordinate system fixed to the system of mass points which is described by $G_{\mu}(\boldsymbol{x})$. 
2. "Rotation of $G_{\mu}(\boldsymbol{x})$ " means: Rotation of the coordinate system fixed to the system of mass points which is described by $G_{\mu}(\boldsymbol{x})$.

Mathematically it will be represented by an operator $R\left(\vartheta_{\mu}, \varphi_{\mu}, \psi_{\mu}\right)$ which acts upon $G_{\mu}(\boldsymbol{x})$ and is defined by

$$
R\left(\vartheta_{\mu}, \varphi_{\mu}, \psi_{\mu}\right) \circ G_{\mu}(\boldsymbol{x}):=\sum_{\nu} P\left(\boldsymbol{x}-\boldsymbol{x}_{\mu \nu}^{\prime}\right)
$$

with

$$
\boldsymbol{x}_{\mu \nu}^{\prime}=A\left(\vartheta_{\mu}, \varphi_{\mu}, \psi_{\mu}\right) \boldsymbol{x}_{\mu v} .
$$

$A\left(\vartheta_{\mu}, \varphi_{\mu}, \psi_{\mu}\right)$ is the rotation matrix corresponding to $R\left(\vartheta_{\mu}, \varphi_{\mu}, \psi_{\mu}\right)$ determined by the Euler angles $\vartheta_{\mu}$, $\varphi_{\mu}, \psi_{\mu}$ acting on $\boldsymbol{x}_{\mu \nu}$. The axes of rotation are passing through the origin of the coordinate system.

\section{Definition of a Rotationally Ordered Distribution}

Since $g(r)$ is independent of $\vartheta$ and $\varphi$ and $R_{\mu}$ only rotates $G_{\mu}$ it follows:

$$
g(r)=\frac{1}{N \varrho_{0}}\left\langle\sum_{\mu}^{N} R_{\mu} \circ G_{\mu}(\boldsymbol{x})\right\rangle_{\vartheta, \varphi} .
$$

The $R_{\mu}$ can now be chosen in such a way as to partly remove the rotational disorder of a structure. In a crystalline powder, for example, the $R_{\mu}$ will be chosen such that equivalent lattice directions are aligned parallel to each other.

The mathematical conditions for this alignement will now be explained, starting from two different $G_{\mu}$ called for example $G_{\alpha}$ and $G_{\beta}$, which belong to two different ideal stressless microcrystals of a powder at $0 \mathrm{~K}$, whose atoms don't move. Parallel orientation of these two microcrystals can now be achieved by postulating that the three Eulerian angles $\vartheta_{\beta}, \varphi_{\beta}, \psi_{\beta}$, which determine the orientation of $G_{\beta}$, are varied until the overlap integral

$$
\begin{aligned}
& O\left(\vartheta_{\beta}, \varphi_{\beta}, \psi_{\beta}\right) \\
& \quad=\int G_{\alpha}(\boldsymbol{x}) \cdot\left(R\left(\vartheta_{\beta}, \varphi_{\beta}, \psi_{\beta}\right) \circ G_{\beta}(\boldsymbol{x})\right) \mathrm{d} V
\end{aligned}
$$

reaches a maximum.

In this case $O\left(\vartheta_{\beta}, \varphi_{\beta}, \psi_{\beta}\right)$ will be zero except for certain $\vartheta_{\beta}, \varphi_{\beta}, \psi_{\beta}$. Apart from a normalization constant the overlap integral (11) will be given by $P\left(\vartheta_{\beta}-\vartheta_{\beta_{0}}, \varphi_{\beta}-\varphi_{\beta_{0}}, \psi_{\beta}-\psi_{\beta_{0}}\right)$ near to its maximum. Equation (11) can also be written as

$$
\begin{aligned}
O_{\alpha \beta}(\boldsymbol{\vartheta}, \boldsymbol{\varphi}, \boldsymbol{\psi})= & \int\left[R\left(\vartheta_{\alpha}, \varphi_{\alpha}, \psi_{\alpha}\right) \circ G_{\alpha}(x)\right] \\
& \times\left[R\left(\vartheta_{\beta}, \varphi_{\beta}, \psi_{\beta}\right) \circ G_{\beta}(x)\right] \mathrm{d} V
\end{aligned}
$$

with

$$
\boldsymbol{\vartheta}=\left(\begin{array}{c}
\vartheta_{1} \\
\vartheta_{2} \\
\cdot \\
\cdot \\
\dot{\vartheta_{N}}
\end{array}\right), \quad \boldsymbol{\varphi}=\left(\begin{array}{c}
\varphi_{1} \\
\varphi_{2} \\
\cdot \\
\cdot \\
\dot{\varphi}_{N}
\end{array}\right), \quad \boldsymbol{\psi}=\left(\begin{array}{c}
\psi_{1} \\
\psi_{2} \\
\cdot \\
\cdot \\
\cdot \\
\psi_{N}
\end{array}\right) \text {. }
$$

$\vartheta_{1}=\varphi_{1}=\psi_{1}=0$ can arbitrarily be chosen since a rotation of all $G_{\mu}$ by the same angles does not change the value of the overlap integral.

Let us now consider all $G_{\mu}$ of the powder. As Eq. (12) defines the pairwise overlap integrals, we can align all $G_{\mu}$ by the condition

$$
O\left(\boldsymbol{\vartheta}_{0}, \boldsymbol{\varphi}_{0}, \boldsymbol{\psi}_{0}\right)=\max _{(\boldsymbol{\vartheta}, \boldsymbol{\varphi}, \boldsymbol{\Psi}}\left\{\sum_{\mu}^{N} \sum_{\nu}^{N} O_{\mu \nu}(\boldsymbol{\vartheta}, \boldsymbol{\varphi}, \boldsymbol{\psi})\right\}
$$

with $\vartheta_{1}=\varphi_{1}=\psi_{1}=0$.

Equation (14) means that one has to rotate synchronously all $G_{\mu}(\mu>1)$ until a maximum overlap of the $G_{\mu}$ with each other is reached. This maximum pairwise overlap defines the $3 \mathrm{~N}$-dimensional vector $\left(\boldsymbol{\vartheta}_{0}, \boldsymbol{\varphi}_{0}, \boldsymbol{\Psi}_{0}\right)$. In the case of nonideal structures Eq. (14) remains unchanged but Eq. (12) has to be modified, since an overlap between different $G_{\mu}$ can now only be expected within certain local fluctuation volumina. In the case of crystalline powder with thermal distortions for example, such an overlap can only be expected if the pointfunctions in the $G_{\mu}$ are smeared over a region as large as the oszillation volume of the atoms.

Thus instead of Eq. (12) we define

$$
\begin{gathered}
O_{\mu \nu}(\boldsymbol{\vartheta}, \boldsymbol{\varphi}, \boldsymbol{\Psi})=\int_{V_{\mathrm{s}}}\left[\left\{R\left(\vartheta_{\mu}, \varphi_{\mu}, \psi_{\mu}\right) \circ \widehat{\left.G_{\mu}(\boldsymbol{x})\right\} L}(\boldsymbol{x})\right]\right. \\
\times\left[\left\{R\left(\vartheta_{v}, \varphi_{v}, \psi_{v}\right) \circ \widehat{\left.G_{v}(\boldsymbol{x})\right\} L}(\boldsymbol{x})\right] \mathrm{d} V .\right.
\end{gathered}
$$

The functional connection

$$
\widehat{f g}=\int f(y) g(x-y) \mathrm{d} V_{y}
$$

is the convolution product of the functions $f$ and $g$. $L(\boldsymbol{x})$ is the LOVE (local overlap)-function which smears out the $P$-functions, contained in the $G_{\mu}$, over a finite region, whose size is in the order of magnitude of the statistical fluctuation. $V_{\mathrm{S}}$ is a short range order volume which has to be introduced in order to exclude the influence of large distance density fluctuations. After having rotated the $G_{\mu}$ until they have maximum overlap with each other according to Eq. (15) and (14), we call

$$
R_{\mu}=R\left(\vartheta_{\mu_{0}}, \varphi_{\mu_{0}}, \psi_{\mu_{0}}\right)
$$


and finally get the rotationally ordered distribution (ROD)-function

$$
g^{+}(\boldsymbol{x})=\frac{1}{N \varrho_{0}} \sum_{\mu}^{N} R_{\mu} \circ G_{\mu}(\boldsymbol{x}) .
$$

This function describes the mean threedimensional short range order as well in a polycrystalline as in an "amorphous" material. Naturally such a function cannot directly be determined from $g(r)$. But there exists the possibility to calculate rotationally ordered distributions from models and then compare the angular average $\left\langle g^{+}(\boldsymbol{x})\right\rangle_{\vartheta}, \varphi$ with experimental $g(r)$. This method has successfully been used in the case of liquid lead ${ }^{2}$. There it was possible to calculate a special ROD at least in a part of space with the help of a convolution polynom based on the theory of paracrystals ${ }^{1}$. Another way is to take the density distribution from molecular dynamics or Monte Carlo calculations and compute the ROD-function in order to see directly what is left of a structure in the liquid.

\section{Calculation of the ROD-Function from Molecular Dynamics Data}

Equation (15) demands to determine the maximum of the sum of the overlap integrals while varying the $3 N$-dimensional vector $(\boldsymbol{\vartheta}, \boldsymbol{\varphi}, \boldsymbol{\psi})$.

Since such a calculation would take to much time, we decided to perform a simpler calculation.

The following procedure was executed by the computer.

1. Take one of the atoms (e.g. $\mu$ ) as origin and store the values of $G_{\mu}$ into a threedimensional field, each field element representing a volume element in space.

2. Take another atom, e.g. $\mu+1$, as origin and rotate the relative position vector $\boldsymbol{x}_{v, \mu+1}=\boldsymbol{x}_{v}-\boldsymbol{x}_{\mu+1}$ of all atoms $v$ which fulfill $\left|\boldsymbol{x}_{v}-\boldsymbol{x}_{\mu+1}\right|<r_{\mathrm{s}}$ [radius of short range sphere $V_{\mathrm{S}}$ in Eq. (15)] through the three Euler angles in steps of $\Delta \vartheta, \Delta \varphi$ and $\vartheta \psi$. For each rotational step the sum of the local overlapintegrals with the stored coordinates has to be determined. That means that the values of those field elements from the threedimensional field (see point 1) are added up, whose corresponding positional vectors $\boldsymbol{r}_{\mathrm{F}}$ fulfill $\left|\boldsymbol{x}_{\mathrm{F}}-\boldsymbol{x}_{(\mu+1) v}^{\prime}\right|<r_{\mathrm{L}}\left(r_{\mathrm{L}}\right.$ radius of the LOVE-sphere), where $\boldsymbol{x}_{v, \mu+1}$ is calculated according to Equation (9). Figure 1 shows schematically the essential feature of this procedure.

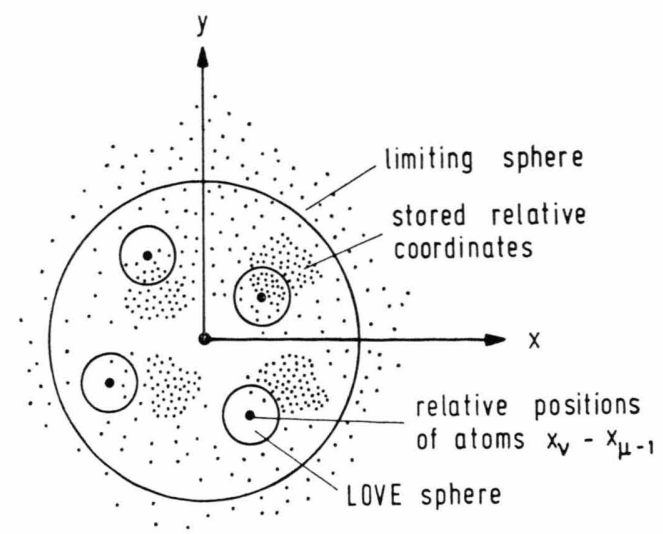

Fig. 1. Twodimensional example for the determination of the rotationally ordered distribution function.

3. After determining in this way the "maximum overlap angles" $\vartheta_{\mu+1,0}, \varphi_{\mu+1,0}, \psi_{\mu+1,0}$ and the corresponding operator $R_{\mu+1}$, according to definition (17) one calculates $R_{\mu+1} \circ G_{\mu+1}$ and adds the value 1 into each field element whose corresponding volume element encloses one of the vectors $\boldsymbol{x}_{(\mu+1) v}^{\prime}$ [see Equation (9)].

Now point 2 . and 3 . have to be repeated for all other atoms $\mu+2, \mu+3, \ldots$ etc.

Apparently this method gives more weight to the first atoms since their environments will be taken as reference systems for all following atoms.

The deviations from the "true" ROD [Eqs. (15), (17), (18)] can be diminished however by performing several runs with different ions as starting reference and averaging after a proper alignment.

As a test substance we chose molten $\mathrm{KCl}$, because the molecular dynamics calculations for this substance had already been performed by one of us ${ }^{3}$.

We used the atomic positions of 216 ions which are in a box of sidelength $20 \AA$ at six different times.

The time between two of these snapshots was $1.2 \cdot 10^{-12} \mathrm{sec}$. The pair correlation function for the $\mathrm{K}^{+}-\mathrm{Cl}^{-}$has a first maximum at about $r=3 \AA$, while the pair correlation functions for the $\mathrm{K}^{+}-\mathrm{K}^{+}$and the $\mathrm{Cl}^{-} \cdot \mathrm{Cl}^{-}$have their first maximum at about $r=4.7 \AA$.

For the calculations the following conditions were chosen:

The threedimensional field was rastered into 8000 cubes of sidelength $1 \AA$ which therefore represents the resolution power of our analysis. Scanning of the three Euler angles was performed in steps of $\pi / 10$. 
For the radius of the LOVE sphere we chose a value of $1.5 \AA$.

Two kinds of calculations were performed.

The first one took into account only the $\mathrm{K}^{+}-\mathrm{K}^{+}$ and $\mathrm{Cl}^{-}-\mathrm{Cl}^{-}$correlation. The second treated the melt as if it were mono-atomic, that means it included also the $\mathrm{K}^{+}-\mathrm{Cl}^{-}$correlations. In the first case $r_{\mathrm{s}}=6 \AA$, in the second $r_{\mathrm{s}}=4.5 \AA$ was chosen.

\section{1. $\mathrm{K}^{+}-\mathrm{K}^{+}$and $\mathrm{Cl}^{-}-\mathrm{Cl}^{-}$correlation}

Three runs were performed in this case, each starting with a different ion, that means with a different $G_{\mu}$ as the first reference system. This was done in order to find out how strong the result is influenced by the sequence chosen. Each run evaluated the ionic positions of the 6 time steps. The ROD's of the three runs were rotated against each other until they all had the same orientation. In order to smoothen the ROD's additionally we added into each field element the contents of the six neighbouring field elements which touched its cube faces, and finally we averaged over the three runs.

Now the angular density fluctuation at $r=4.7 \AA$ (the first maximum of the $\mathrm{K}^{+}-\mathrm{K}^{+}$and $\mathrm{Cl}^{-}-\mathrm{Cl}^{-}$pair correlation function) was investigated. Since the surface element $\mathrm{d} F$ of a sphere with radius $r$ is given by

$$
\mathrm{d} F=r^{2} \sin \vartheta \mathrm{d} \vartheta \mathrm{d} \varphi
$$

( $\vartheta, \varphi$ inclination- and azimuthal angle respectively), we increased in our spherical projection the number of raster points for $\varphi$ by $\sin \vartheta$ so that all raster points represent equally sized areas. $g^{+}(r, \vartheta, \varphi)$ projected in that way is shown in Figure 2.

The two parts of Fig. 2 represent two halves of one spherical shell. On the connection circle at $\varphi= \pm \pi / 2$ the density values are equal for both halves.

The $6 \cdot 2 \cdot 108=1296$ origins are positioned in the center of the sphere. Lines were drawn around all maxima of the angular density distribution. Twelve maxima can be seen (the broken line encircles a region which is the extension of a maximum at $r=6.5 \AA$ ).

In order to show the dependance of the sequence of calculations, the corresponding ROD's of the three runs are shown in Figure 3.

Concerning the coarse raster used during the calculations the three functions agree fairly well

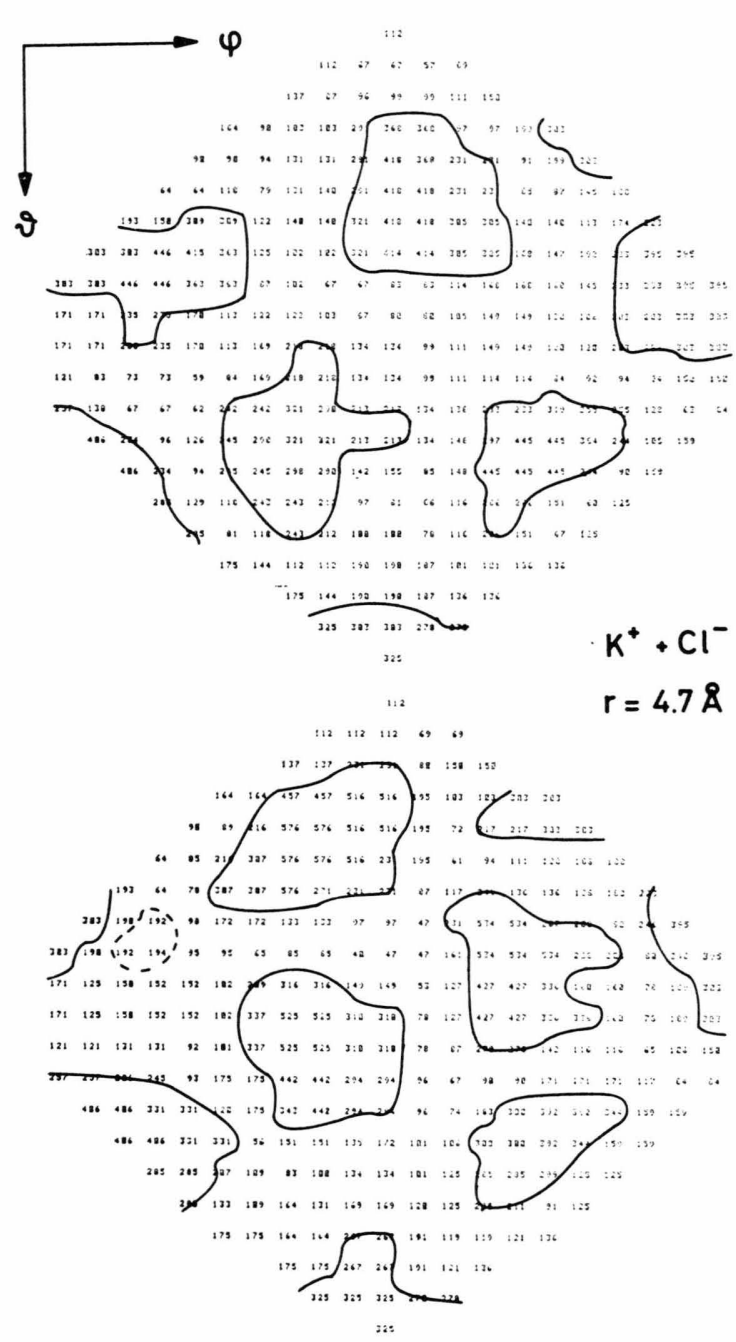

Fig. 2. Values of the ROD for the sum of the $\mathbf{K}^{+}-\mathbf{K}^{+}$and the $\mathrm{Cl}^{-} \cdot \mathrm{Cl}^{-}$correlation on a spherical shell at distance $r=$ 4.7 $\AA$ from the origin of the coordinate system. This is the average of the three properly aligned runs, which are shown in Fig. 3.

with each other. For a reliable calculation of the standard deviation, however, one would need more runs. Since a quantitative evaluation of the ROD is not the aim of this work, and since each run takes a lot of time, we want to leave that question for a later work.

\section{Total Correlation}

In this case all ionic positions were used. Accord. ing to the investigation of the $\mathrm{K}^{+}-\mathrm{K}^{+}$correlation the essential features of the structure are already visible after one run. So only one run was performed. 

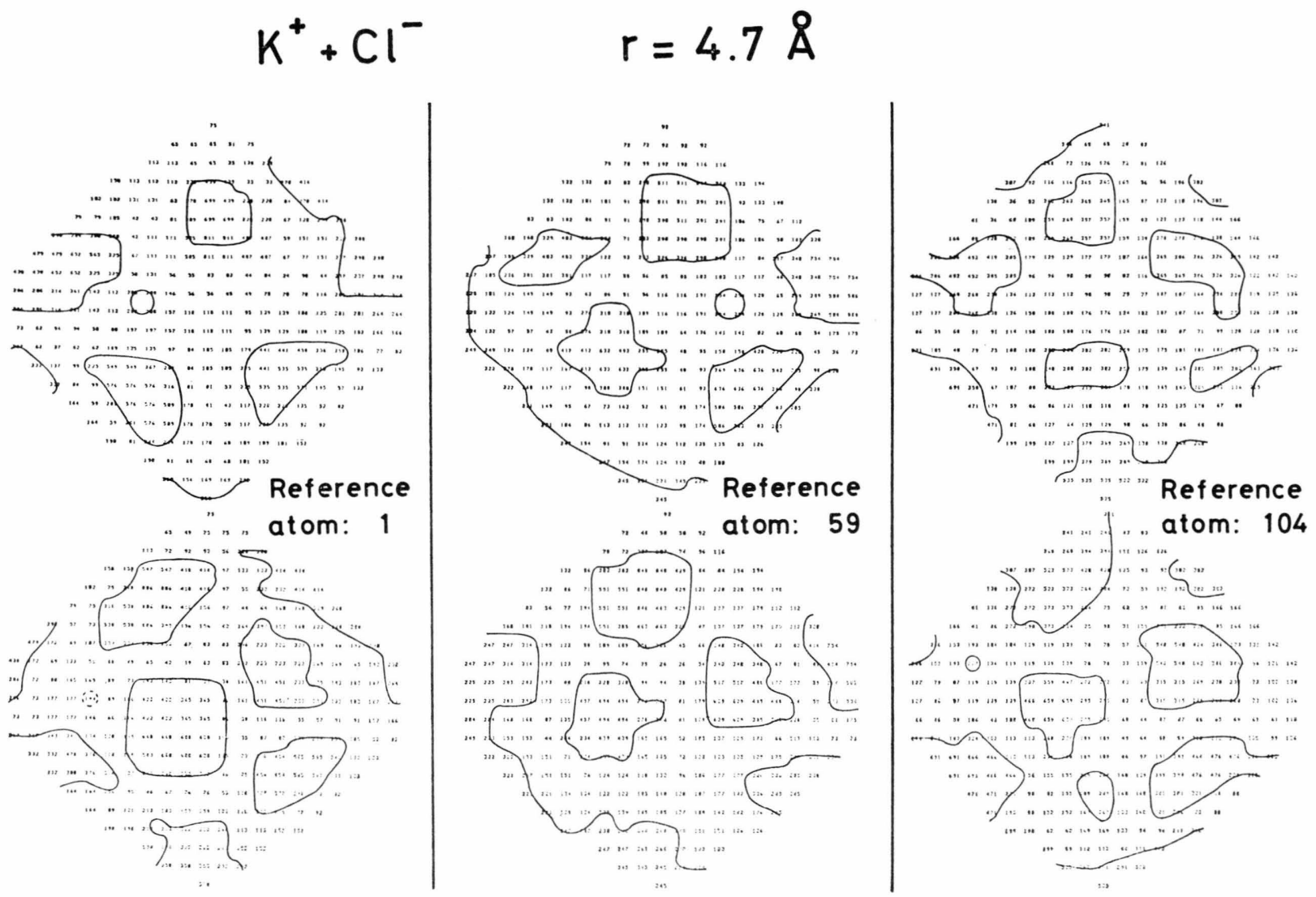

Fig. 3. Three aligned ROD's for the $\mathrm{K}^{+}-\mathrm{K}^{+}$and $\mathrm{Cl}^{-}-\mathrm{Cl}^{-}$correlation on a spherical shell at distance $r=4.7 \AA$ from the reference atoms. Each of the three calculations started with a different atom.

The projection of the ROD at $r=3.5 \AA$ is shown in Figure 4.

$3.5 \AA$ was chosen because at this distance all peaks can best be seen at the same time. Naturally there is not only an angular fluctuation of the ion positions but also a distance fluctuation, and according to the paracrystalline analysis of liquids 4 the mean distance of nearest neighbours is usually slightly larger than the most probable distance. The main maxima are schematically encircled.

\section{Interpretation}

Let us first consider the number of nearest neighbour peaks produced by the two kinds of calculations. In the first case this number is 12 , in the second case it is 6 .

It should be mentioned that these numbers are not identical with the average numbers of nearest neighbours, the socalled coordination numbers. In order to find the coordination numbers one would have to integrate over the nearest neighbour peaks, which however would require the possibility to separate the nearest neighbour-statistics from the second nearest, third nearest, and so on. But such a separation is only possible on the basis of a suitable model.

The next point is the interpretation of the structure revealed by the position of the maxima of the ROD's. The numbers of peaks suggest to start from the corresponding solid state structures, namely primitiv cubic for the total correlation and face centered cubic (fcc) for the other.

The ideal cubic primitiv environment is shown in Fig. 5 for comparison. Obviously the peak distribution in Fig. 4 can be approximated by a distorted cubic primitiv arrangement. It is not so easy however to see the resemblance between the peak-position-distribution in Fig. 2 and a fccsurrounding. Therefore the ROD of Fig. 2 is redrawn 


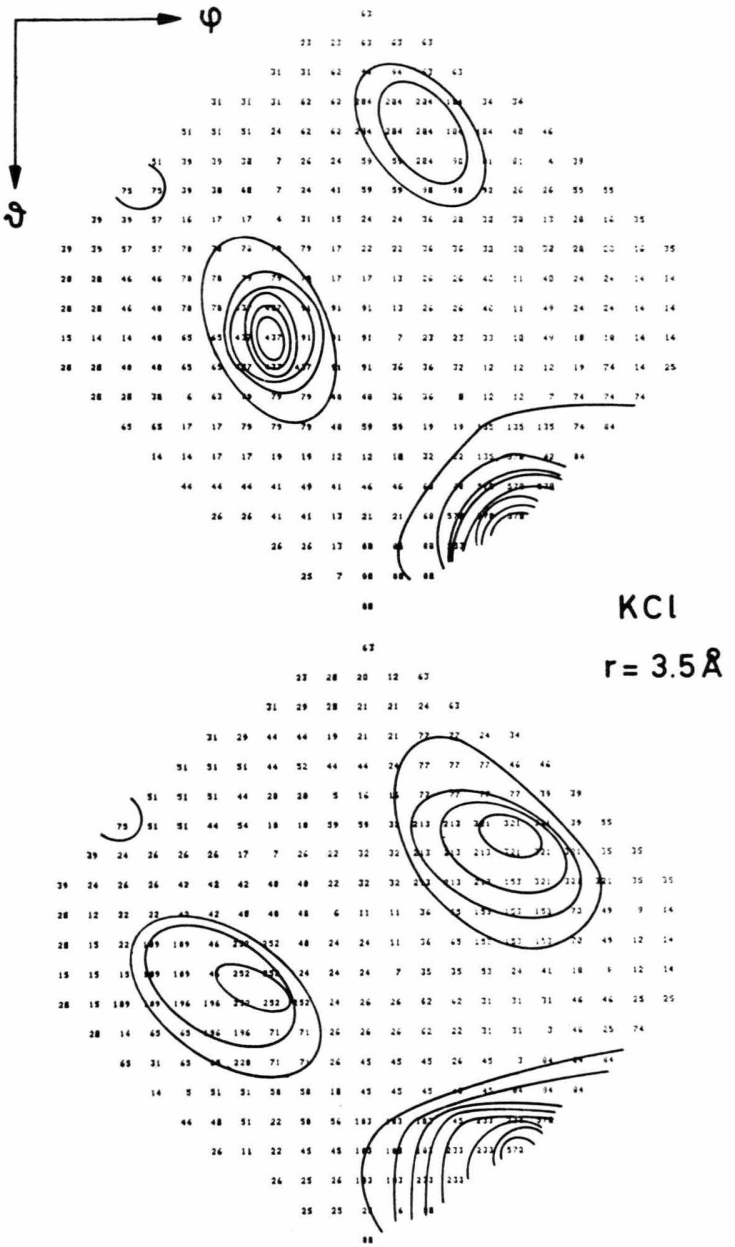

Fig. 4. ROD for the correlation between all atoms of the $\mathrm{K}-\mathrm{Cl}$ melt. The values given are those on a spherical shell at distance $r=3.5 \AA$ from the reference atoms.

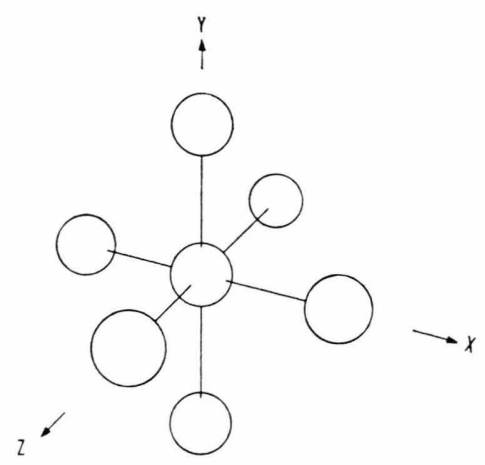

Fig. 5. Perspective drawing of a cubic arrangement of atoms around an atom in the origin of the coordinate system for comparison with Figure 4.

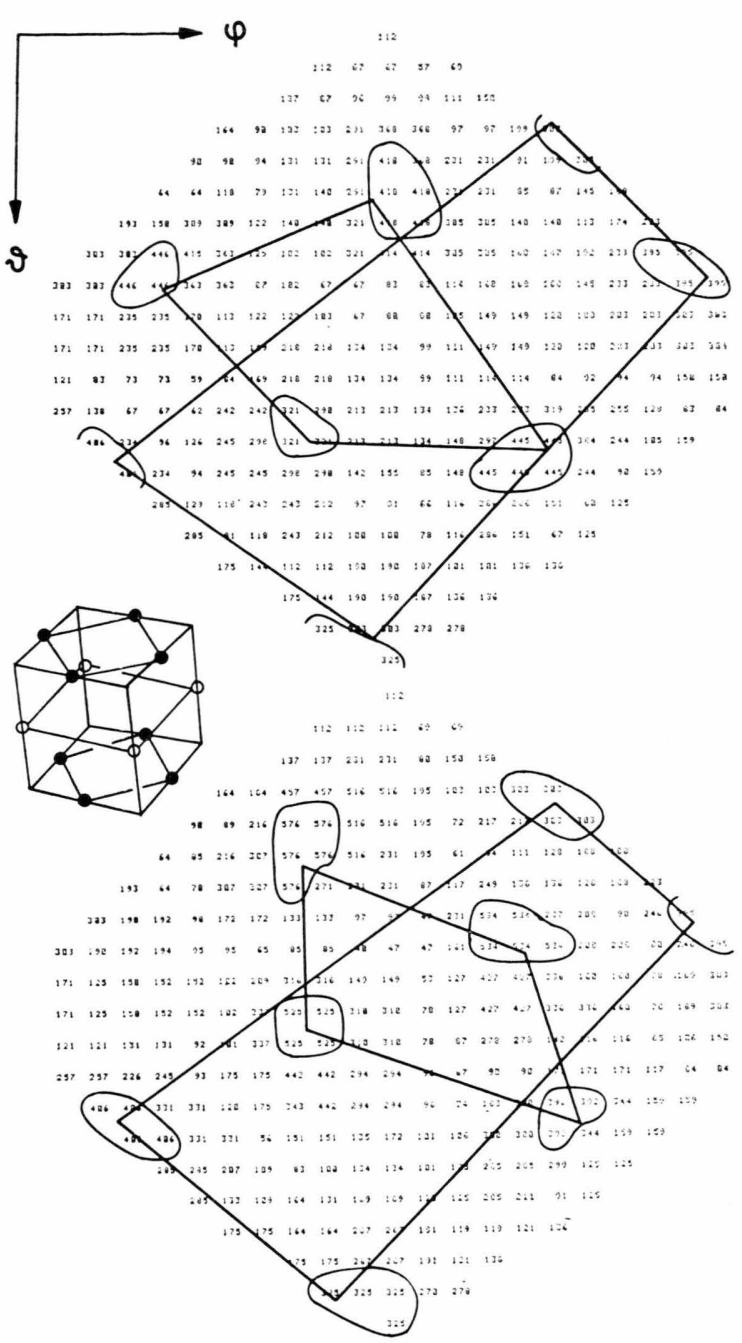

Fig. 6. Positions of the maxima of the $\mathrm{K}^{+}-\mathrm{K}^{+}$and $\mathrm{Cl}^{-}-\mathrm{Cl}^{-}$ROD as in Fig. 2, compared to a face centered cubic arrangement of atoms.

in Fig. 6 in comparison with a fcc surrounding. The twelve nearest neighbours can be divided into three groups of four atoms. Each of the groups a, b, and c has a quadratical form and the groups are rotated against each other by $\pi / 4$.

Similar relationships can be seen in the projection of the ROD. Naturally this surrounding is not ideal. Its deviations from the ideal fcc surrounding are such that it can also be described as a distorted icosahedron. A result which fits very well with the conclusions from the paracrystalline analysis of molten lead ${ }^{2}$. 


\section{Conclusions}

The rotationally ordered distribution as defined above gives a threedimensional picture of the mean short range order in amorphous materials. This mean short range order reveales common properties of all microdomains in the material. Such properties are not directly recognizable from the density distribution itself, since there they will be covered by the strong structural fluctuations in the substance. The difference between the density distribution and the ROD is comparable to the difference between electron microscopy and x-ray scattering. Only the ROD averages over less structural properties than scattering experiments. In the case of molten potassium chloride it reveales a mean structure which strikingly resembles the solid state structure. Although these maxima are broadened compared to the solid state distribution, nevertheless the angular density fluctuation in the melt is by no means negligible.

1 R. Hosemann and S. N. Bagchi, Direct Analysis of Diffraction by Matter, North-Holland Publ. Comp., Amsterdam 1962.

2 B. Steffen and R. Hosemann, Phys. Rev. B13, 3232 [1976].
We think that such angular density fluctuations are not a special property of potassium chloride but will occur in all melts with a sufficiently high packing density.

Two main conclusions follow from this analysis:

1. The mean short range order of a melt still contains the rudiments of a suitably distorted lattice. This fact supports the description of the short range structure of a melt as a paracrystalline lattice with the help of a convolution polynomial as proposed by Hosemann ${ }^{1}$ and performed by Steffen and Hosemann ${ }^{3}$.

2. For calculations of short range quantities as for example certain electron wave functions in liquid metals and corresponding effective pair potentials it is necessary to take into account angular density fluctuations. Generally first all other mathematical operations should be performed and then the angular average may be taken.

3 L. Schäfer and A. Klemm, Z. Naturforsch. 31 a, 1068 [1976].

4 G. Willmann and R. Hosemann, Z. Naturforsch. 26a, 1865 [1971]. 